Supporting Information for:

\title{
Compact and Integrated Approach for Advanced End-to-End Pro- duction, Purification, and Aqueous Formulation of Lidocaine Hydrochloride
}

Jean-Christophe M. Monbaliu, ${ }^{\dagger, \ddagger}$ Torsten Stelzer, $^{\dagger, \S}$ Eve Revalor, ${ }^{\dagger} \|$ Nopphon Weeranoppanant, ${ }^{\dagger}$ Klavs F. Jensen, ${ }^{* \dagger}$ and Allan S. Myerson ${ }^{*} \dagger$

${ }^{\dagger}$ Department of Chemical Engineering, Massachusetts Institute of Technology 77 Massachusetts Ave., Cambridge, Massachusetts 02139, United States

${ }^{\ddagger}$ Center for Integrated Technology and Organic Synthesis, Department of Chemistry, University of Liège, B-4000 Liège (Sart Tilman), Belgium

${ }^{\S}$ Department of Pharmaceutical Sciences, University of Puerto Rico, Medical Sciences Campus, San Juan, Puerto Rico, United States

"Department of Chemical and Biomolecular Engineering, Melbourne Medical School, University of Melbourne, Melbourne, 3010 Victoria, Australia

\section{Table of contents}

I. Unit Operations $\quad$ S2

II. Solute-Liquid Equilibrium (SLE) Determination $\quad$ S7

III. Nuclear Magnetic Resonance Spectroscopy (NMR) S9

IV. High Pressure Liquid Chromatography (HPLC) S13

V. Ultrasound Monitoring $\quad$ S14

VI. Molar Ratio S16

$\begin{array}{lll}\text { VII. } & \text { Additional Information Details } & \text { S17 }\end{array}$

$\begin{array}{ll}\text { VIII. References } & \text { S20 }\end{array}$ 


\section{Unit operations}

\section{Reaction crystallizer.}
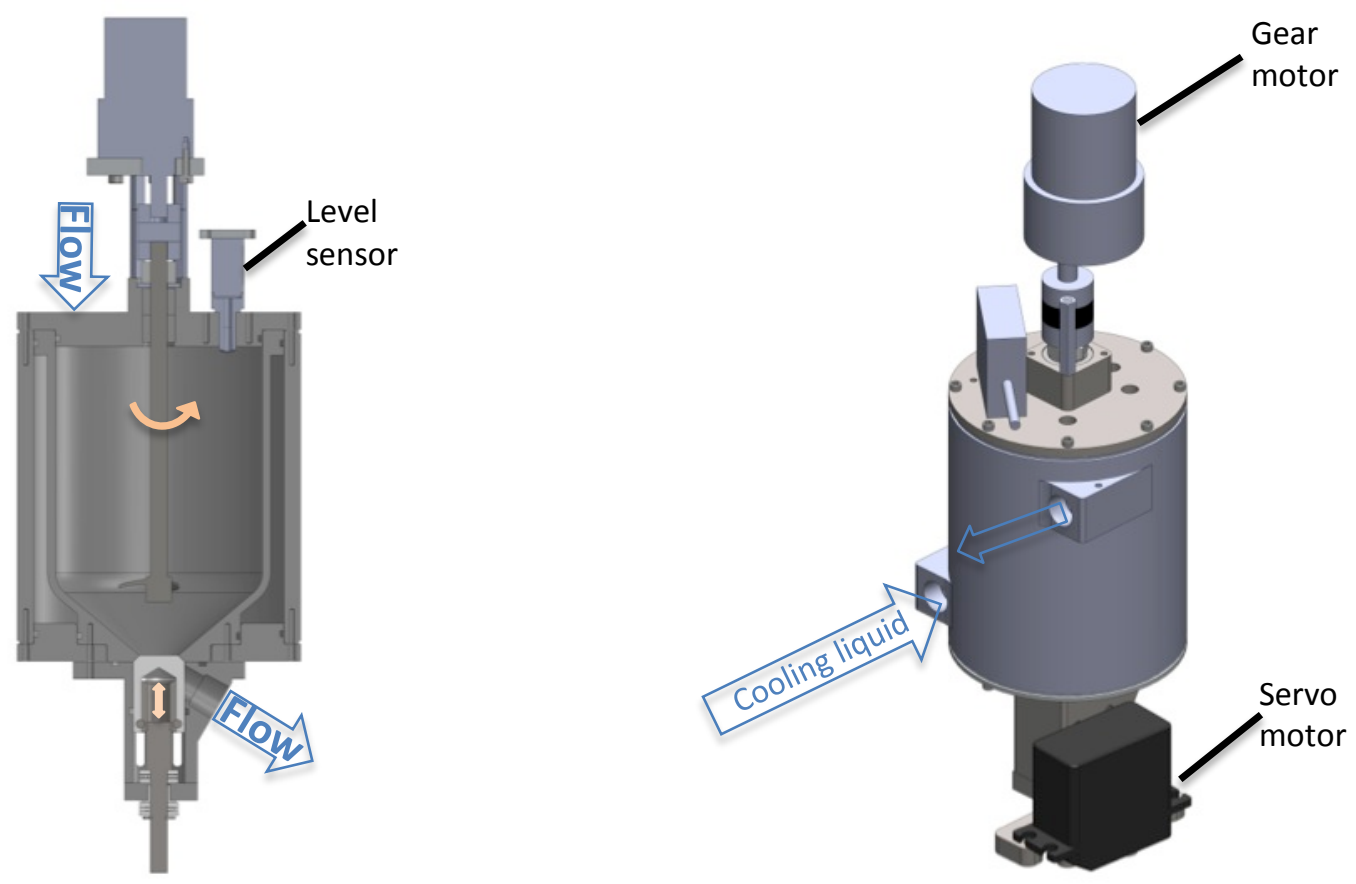

Figure S1. Reactive crystallizer equipped with a bottom drain valve (plunger moving up and down via servo motor as indicated by the orange arrow in the left figure allowing opening and closing of the reaction crystallizer), a PTFE coated marine impeller (rotating via gear motor), and a level sensor. The solution enters the crystallizer through the top cover and drains via the bottom valve (as indicated with the blue arrows in the left figure). The unit is double-jacketed allowing a cooling fluid to circulate around the unit (as indicated by the blue arrows in the right figure). Left shows the cross-section whereas the right shows the $3 \mathrm{D}$ model. The overall unit measures $70 \mathrm{~mm}$ in diameter.

Filtration-drying-dissolution unit. The design of the filtration-drying-dissolution unit (FDD) was modified from a report of Wong et al., ${ }^{1}$ and adapted to our process. The parts were machined out of HDPE for the FDD after the reactive crystallization unit and out of stainless steel 316L for the FDD after the antisolvent cooling crystallization unit (see general process flow chart in Figure 1). The FDDs were mounted in a $45^{\circ}$ angle and operated by distributing the slurry 
on top of sintered 0.5 graded Hastelloy plates (Mott Corporation, USA) while vacuum was applied through the porous plates (Figure S2). The vacuum was enabled through transfer lines to the waste tank connected to a vacuum pump (UN811 KVP, KNF Neuberger, Germany). Once the residual mother liquor was removed, the vacuum was closed by solenoid valves (Type 0127, Christian Bürkert GmbH \& Co. KG, Germany) and wash solvent was pumped into the FDD’s and pulled through the porous plate by opening the valve after washing. The solenoid valves with PEEK (polyether ether ketone) manifolds and FFPM (perfluoro-polymer) sealing material were utilized for all subunits downstream. The subsequent vacuum drying step was initiated by heating the FDD's with a wrapped flexible heater (KH series, Omega Engineering, Inc, USA) controlled by a T-type thermocouple (TJC36 series, Omega Engineering, Inc, USA). Once the drying step was completed, the valve was closed and the solvent was pumped into the FDD's to resuspend the dried solids to be transferred to the subsequent unit by opening the in-house built bottom drain valve (BDV).

Since the footprint of commercial BDVs suitable for slurry transportations were not compatible with the compactness of this pharmaceutical manufacturing platform, compact BDVs were machined and utilized for all subunits downstream. The customized BDVs take advantage of gravity as the driving force for the slurry transportation. BDVs were opened and closed using a plunger driven by a servo motor (pulse width modulation (PWM) controlled). The outlets of the units and BDVs in the downstream section have a diameter of $10 \mathrm{~mm}$ to prevent clogging issues. ${ }^{2}$ 

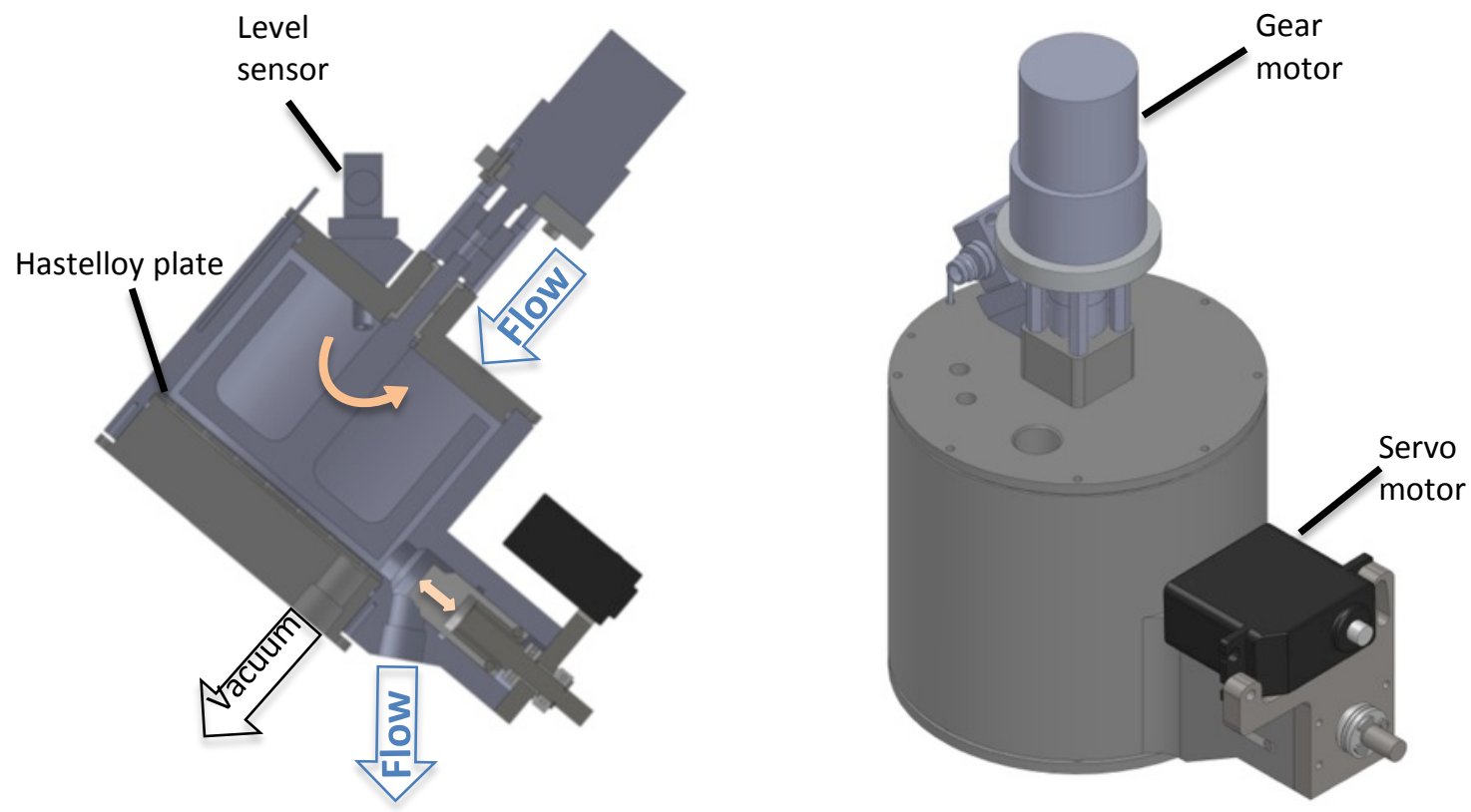

Figure S2. Filtration-drying-dissolution unit (FDD), adapted and modified from Wong et al., ${ }^{1}$ equipped with a bottom drain valve (plunger moving back and forth via servo motor as indicated by the orange arrow in the left figure allowing opening and closing of the FDD), a thermocouple, a porous Hastelloy plate, a PTFE coated anchor stirrer (rotating via gear motor), and a level sensor. The solution enters the FDD through the top cover and exits via the bottom valve (as indicated by the blue arrows in the left figure). The vacuum is applied through the bottom of the FDD underneath the membrane (as indicated by the black arrow in the left figure). The left image shows the cross-section while the right shows the 3D model. ${ }^{2}$ The overall unit measures 100 $\mathrm{mm}$ in diameter. 


\section{Antisolvent cooling crystallizer.}
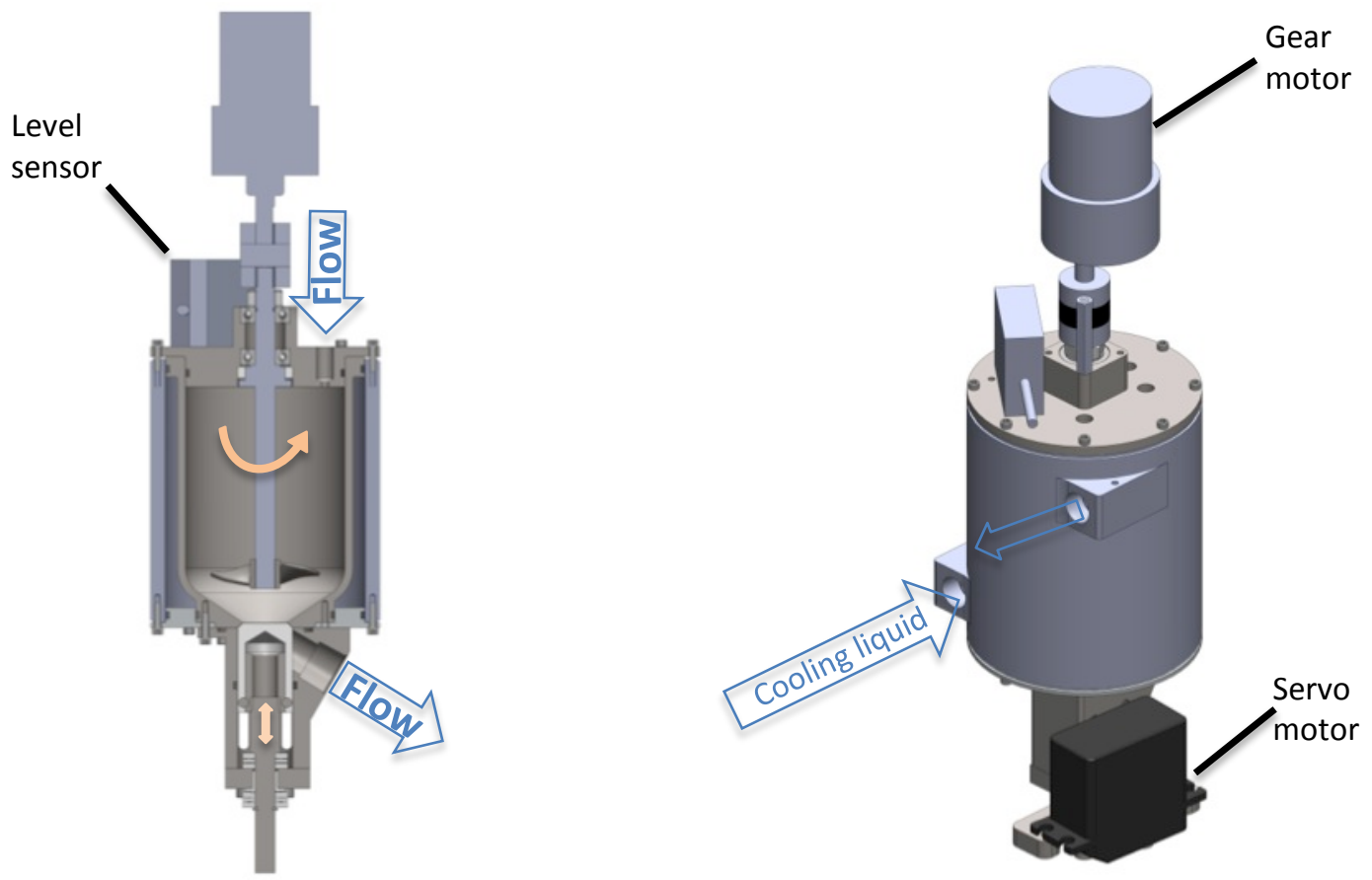

Figure S3. Crystallizer equipped with a bottom drain valve (plunger moving up and down via servo motor as indicated by the orange arrow in the left figure allowing opening and closing of the crystallizer), a PTFE coated marine impeller (rotating via gear motor), and a level sensor. The solution enters the crystallizer through the top cover and drains via the bottom valve (as indicated with the blue arrows in the left figure). The unit is temperature regulated by a cooling liquid flowing around the unit as indicated by the blue arrows in the right figure. Left shows the cross-section whereas the right shows the 3D model. ${ }^{2}$ The overall unit measures $70 \mathrm{~mm}$ in diameter.

Formulation tank. The formulation tank is a $500 \mathrm{~mL}$ stainless steel 316L vessel equipped with a bottom drain valve, an ultrasound sensor (Liquisonic30, SensoTech GmbH), a level sensor (crz15/CU/K2, Microsonic GmbH), and a marine impeller (Figure S4). Here, the concentration and temperature were monitored and adjusted as necessary by addition of a premixed solution and a thermoelectric liquid cooler (LC-035, TE Technology, Inc.), respectively, before the formulated concentrate was drained into the final storage container by opening the bottom drain valve. 

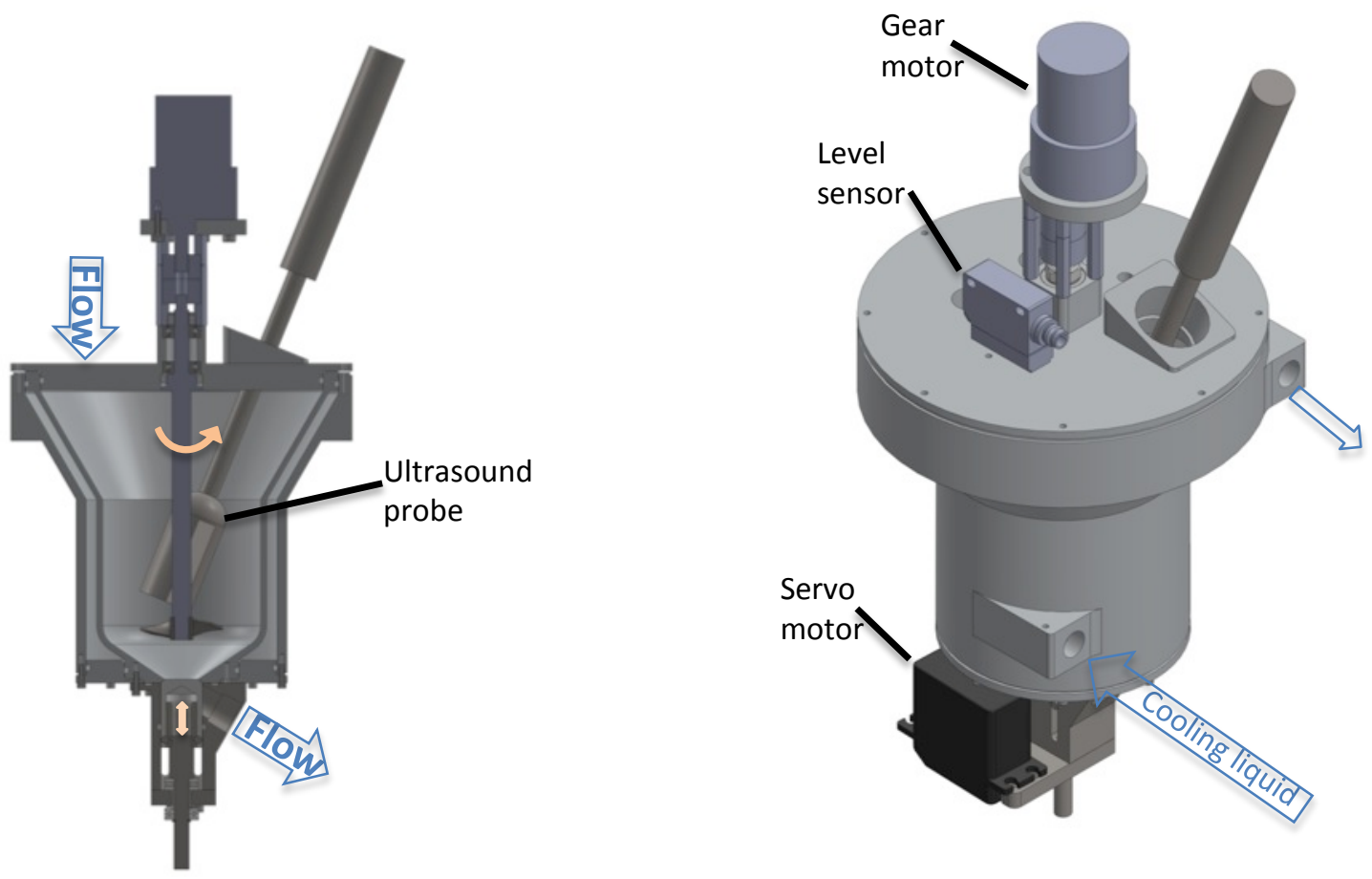

Figure S4. Formulation tank equipped with a bottom drain valve (plunger moving up and down via servo motor as indicated by the orange arrow in the left figure allowing opening and closing of the formulation tank), a ultrasound probe, a marine impeller (rotating via gear motor), and a level sensor. The solution enters the formulation tank through the top cover and drains via the bottom valve on demand (as indicated with the blue arrows in the left figure). The unit is temperature regulated by a cooling liquid flowing around the unit as indicated by the blue arrows in the right Figure. Left shows the cross-section whereas the right shows the 3D model. The overall unit diameter is $100 \mathrm{~mm}$ in the narrow part. 


\section{Solute-Liquid Equilibrium (SLE) Determination}

A Crystal $16^{\mathrm{TM}}$ device (Avantium Tec. BV, The Netherlands) was used to conduct experimental solute-liquid equilibrium (SLE) studies by means of a polythermal dissolution method. ${ }^{3}$ Sealed flasks with a volume of $2 \mathrm{~mL}$ were filled with a predefined amount of lidocaine (5), lidocaine hydrochloride $(\mathbf{5} \cdot \mathrm{HCl})$ and an appropriate solvent. The obtained suspensions were heated under agitation at $300 \mathrm{rpm}$ from 2 to $50^{\circ} \mathrm{C}$. A turbidity probe was utilized for determining the clear points and the corresponding temperatures were recorded.

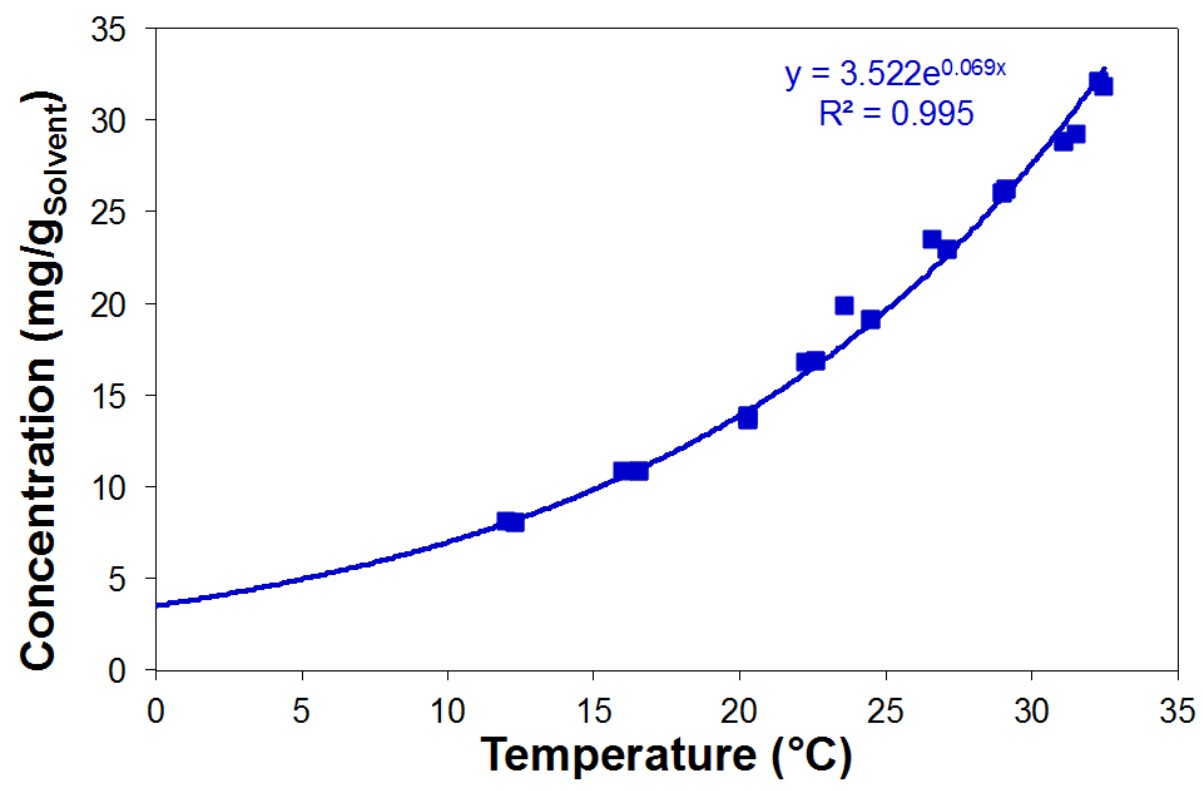

Figure S5. Solute-liquid equilibrium of a reference sample (Sigma-Aldrich) of lidocaine (5) in hexane. 


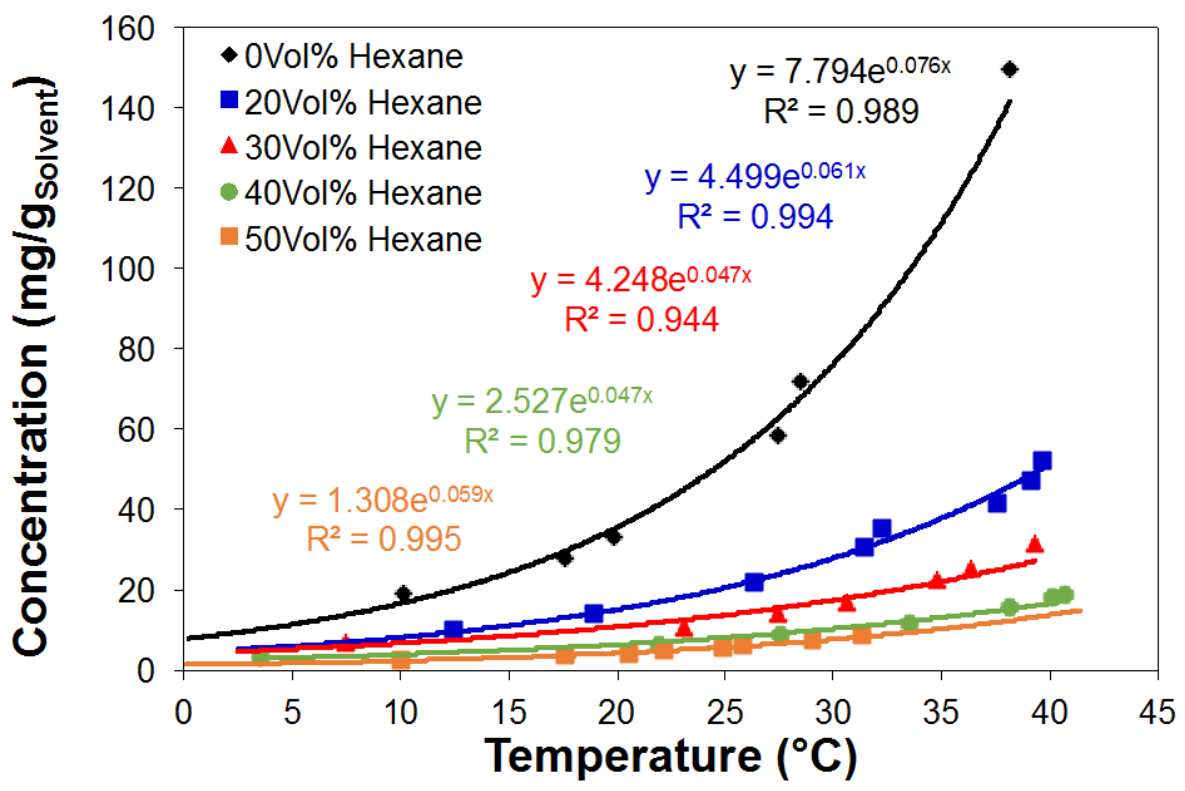

Figure S6. Solute-liquid equilibrium of a reference sample (Sigma-Aldrich) of lidocaine hydrochloride $(5 \cdot \mathrm{HCl})$ in acetone/isopropanol $(96: 4 \mathrm{wt}-\%)$ as a function of antisolvent (hexane) content (Vol\%).

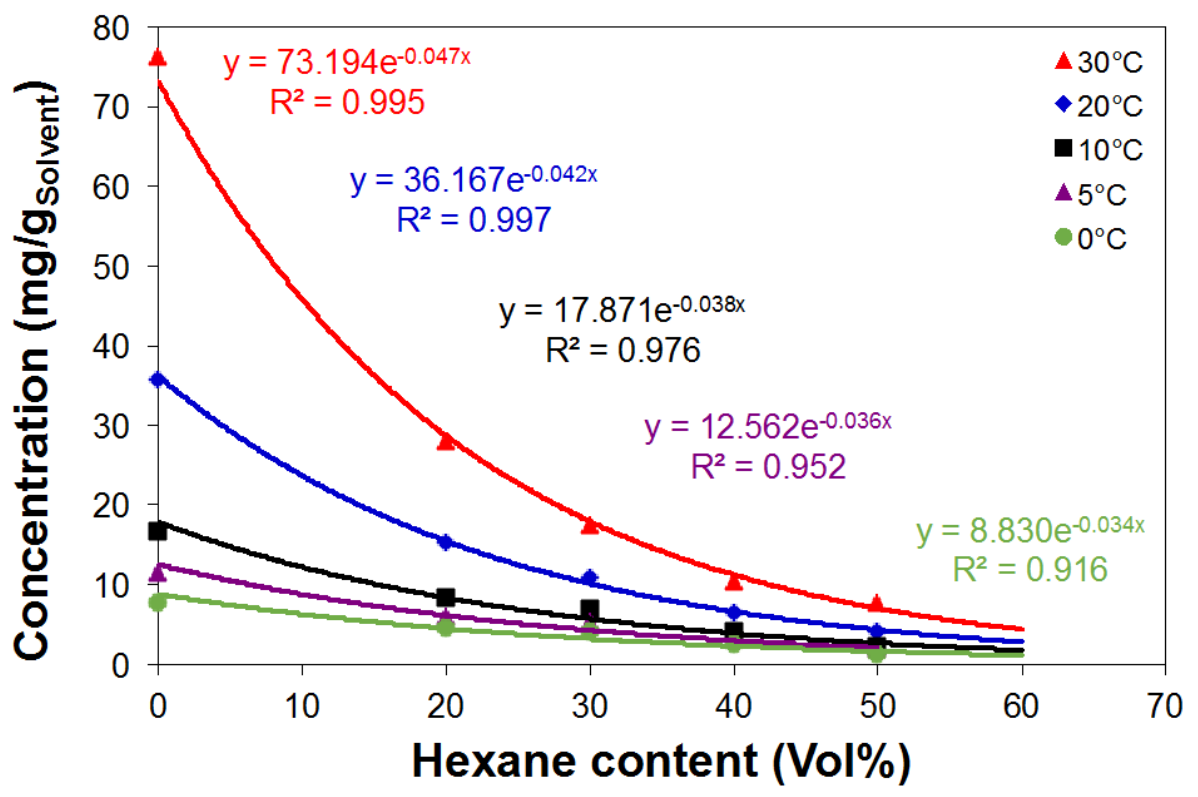

Figure S7. Concentration of a reference sample (Sigma-Aldrich) of lidocaine hydrochloride $(5 \cdot \mathrm{HCl})$ versus antisolvent (hexane) volume fraction $(\mathrm{Vol} \%)$ as a function of temperature $\left({ }^{\circ} \mathrm{C}\right)$. 


\section{Nuclear Magnetic Resonance Spectroscopy (NMR)}

${ }^{1} \mathrm{H}$ NMR spectra were recorded on a Bruker $300 \mathrm{MHz}$ spectrometer at room temperature in $d_{6}$-DMSO, $\mathrm{D}_{2} \mathrm{O}$ or $\mathrm{CDCl}_{3}$. Tetramethylsilane was used as an internal standard when appropriate.

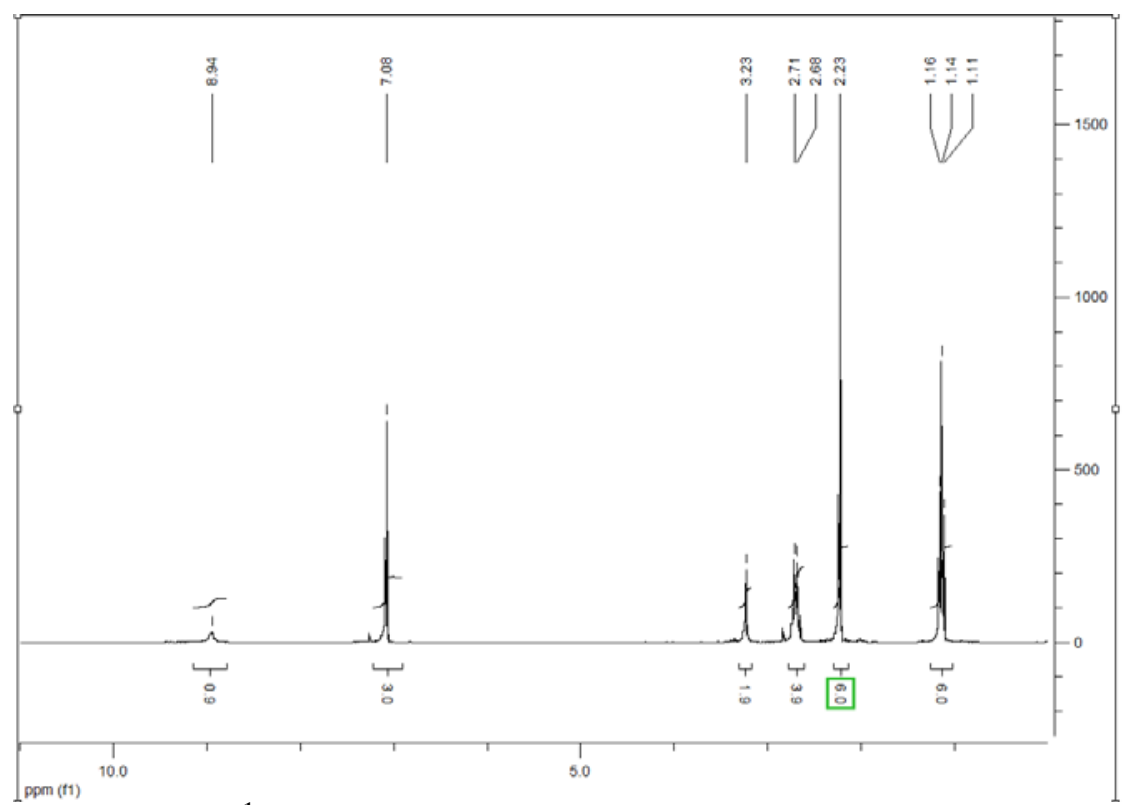

Figure S8. ${ }^{1} \mathrm{H}$ NMR $\left(\mathrm{CDCl}_{3}\right)$ of crude lidocaine (5) after extraction with Method B $\left(\mathrm{NaCl} / \mathrm{NH}_{4} \mathrm{Cl}\right)$.

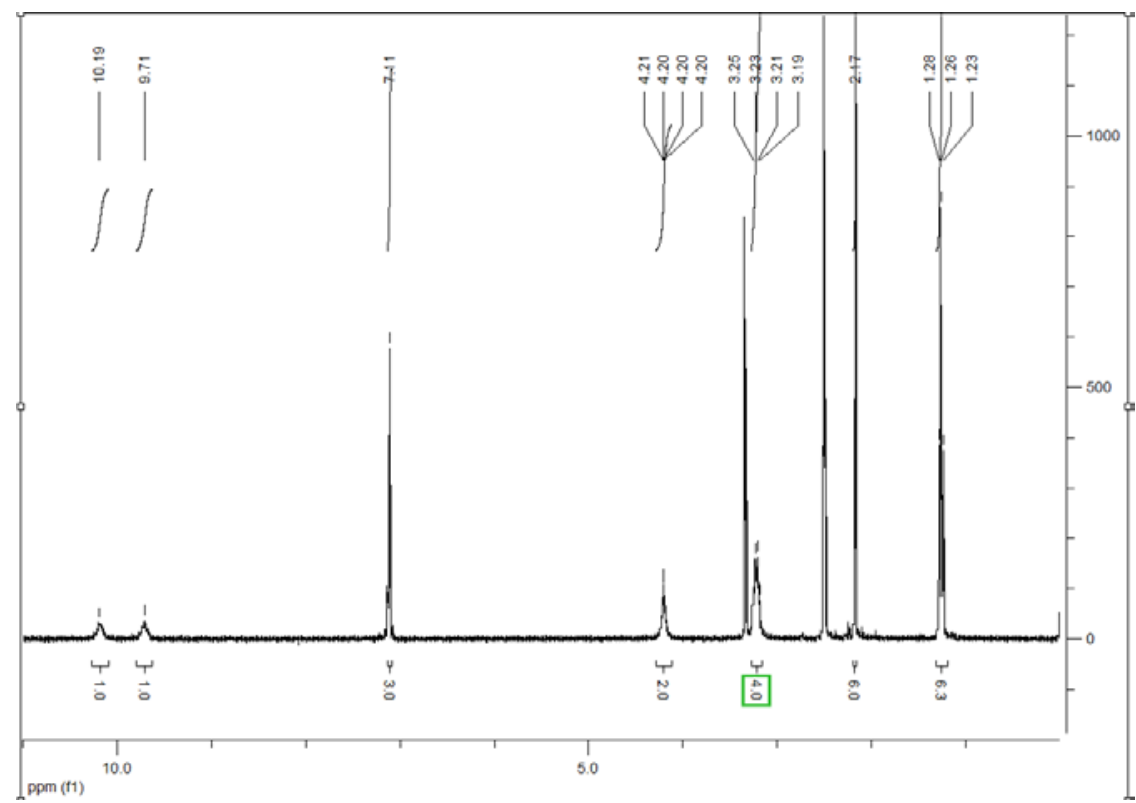

Figure S9. ${ }^{1} \mathrm{H}$ NMR $\left(d_{6}\right.$-DMSO) of commercial (Sigma-Aldrich) lidocaine hydrochloride $(5 \cdot \mathrm{HCl})$. 


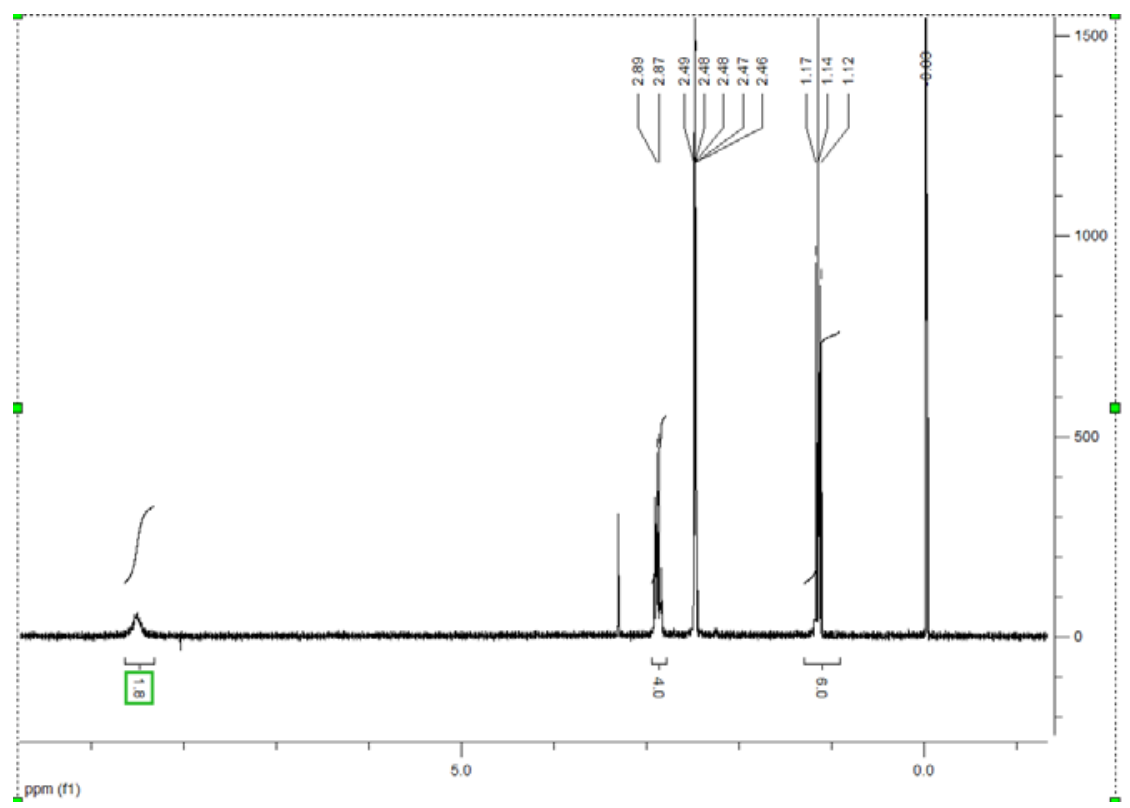

Figure S10. ${ }^{1} \mathrm{H}$ NMR $\left(d_{6}\right.$-DMSO) of diethylamine hydrochloride $(4 \cdot \mathrm{HCl})$.

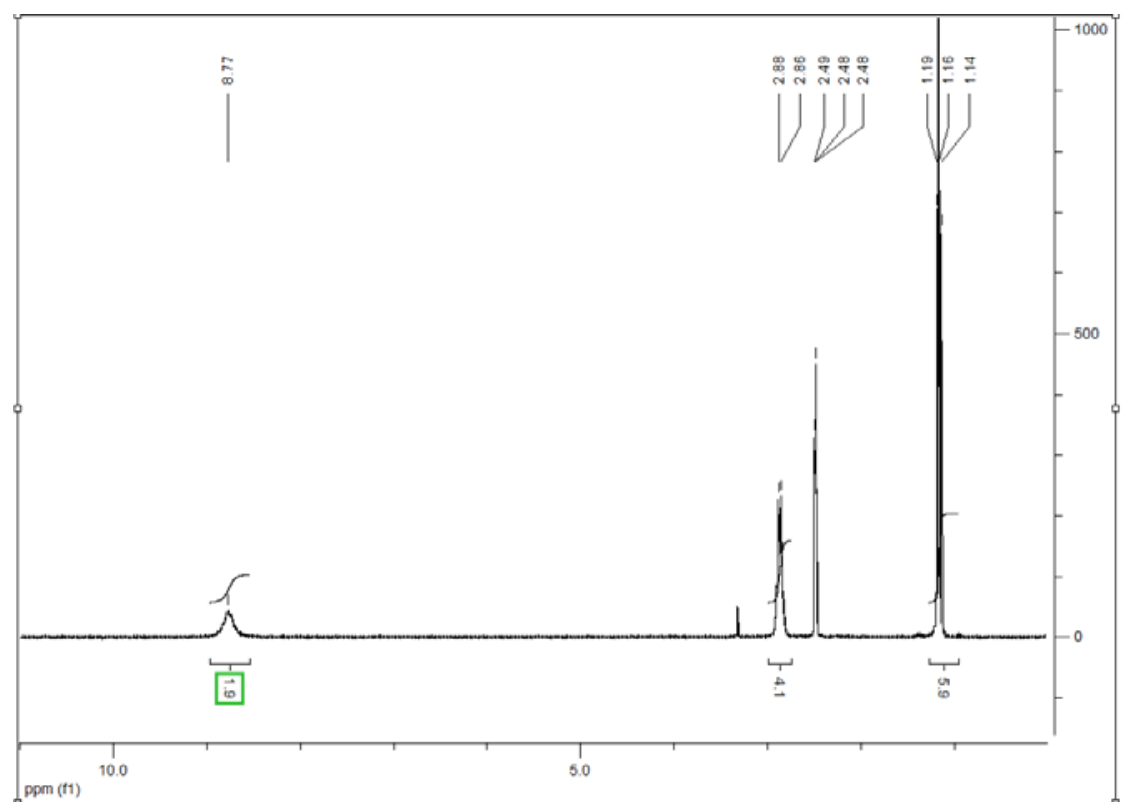

Figure S11. ${ }^{1} \mathrm{H}$ NMR ( $d_{6}$-DMSO) of a solid sample collected in the reaction tank after $60 \mathrm{~min}$ ripening (upstream extraction: $\mathrm{NaCl}$ only, Method A). Analysis revealed the presence of diethylamine hydrochloride $(4 \cdot \mathrm{HCl})$ only. 


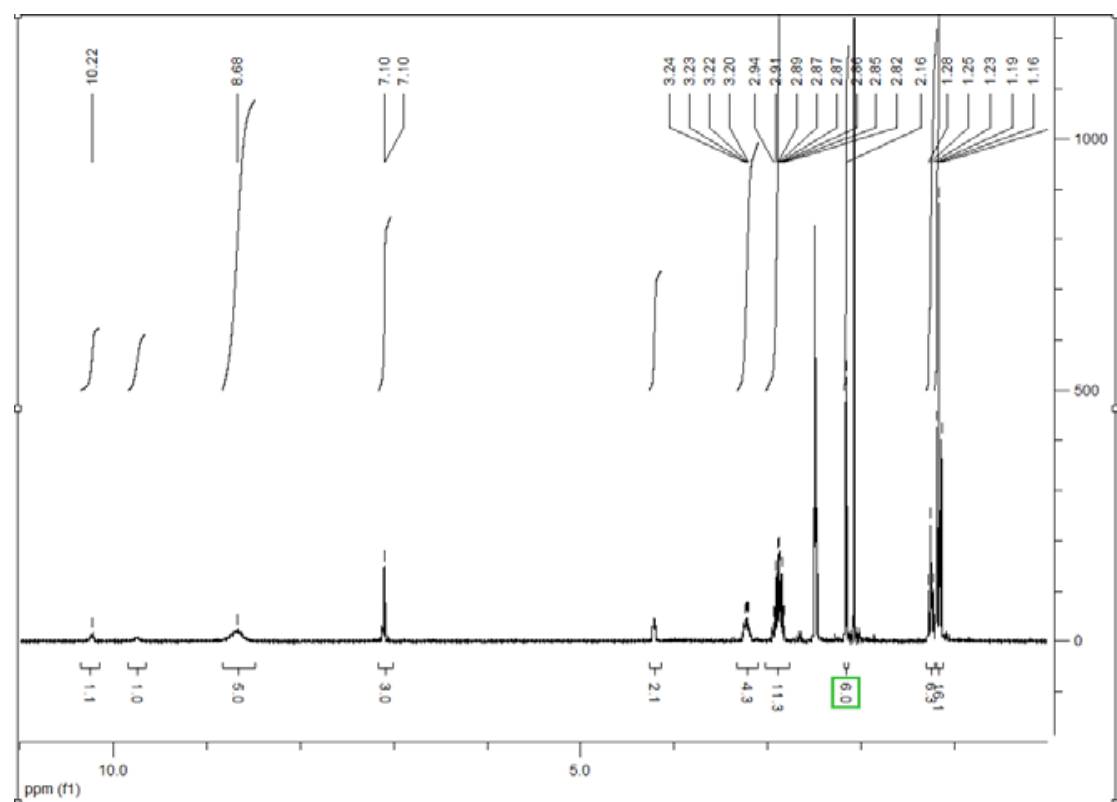

Figure S12. ${ }^{1} \mathrm{H}$ NMR $\left(d_{6}\right.$-DMSO) of a solid sample collected in the reaction tank after $120 \mathrm{~min}$ ripening (upstream extraction: $\mathrm{NaCl}$ only, Method A). Analysis revealed a lidocaine hydrochloride $(\mathbf{5} \cdot \mathrm{HCl}) /$ diethylamine hydrochloride $(\mathbf{4} \cdot \mathrm{HCl})$ 25:75 mixture.

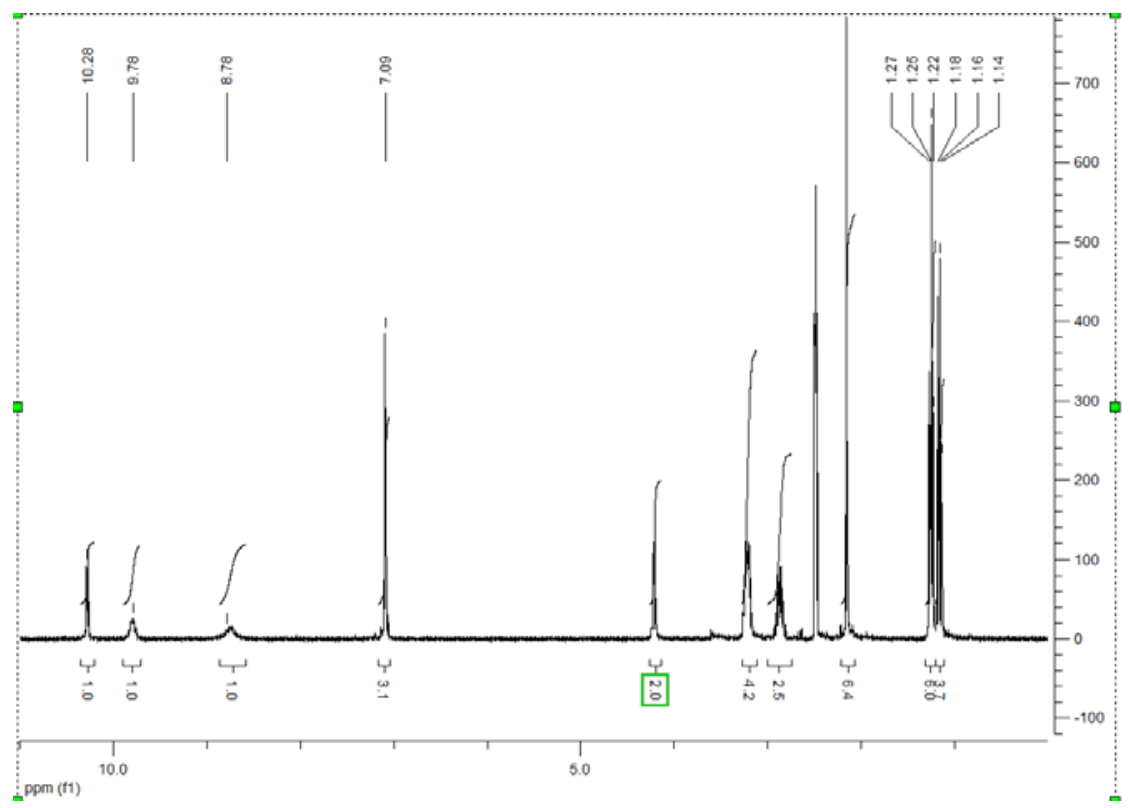

Figure S13. ${ }^{1} \mathrm{H}$ NMR ( $d_{6}$-DMSO) of a solid sample collected in the reaction tank after 13 h ripening (upstream extraction: $\mathrm{NaCl}$ only, Method A). Analysis revealed a lidocaine hydrochloride (5· $\mathrm{HCl})$ / diethylamine hydrochloride $(\mathbf{4} \cdot \mathrm{HCl})$ 61:39 mixture. 


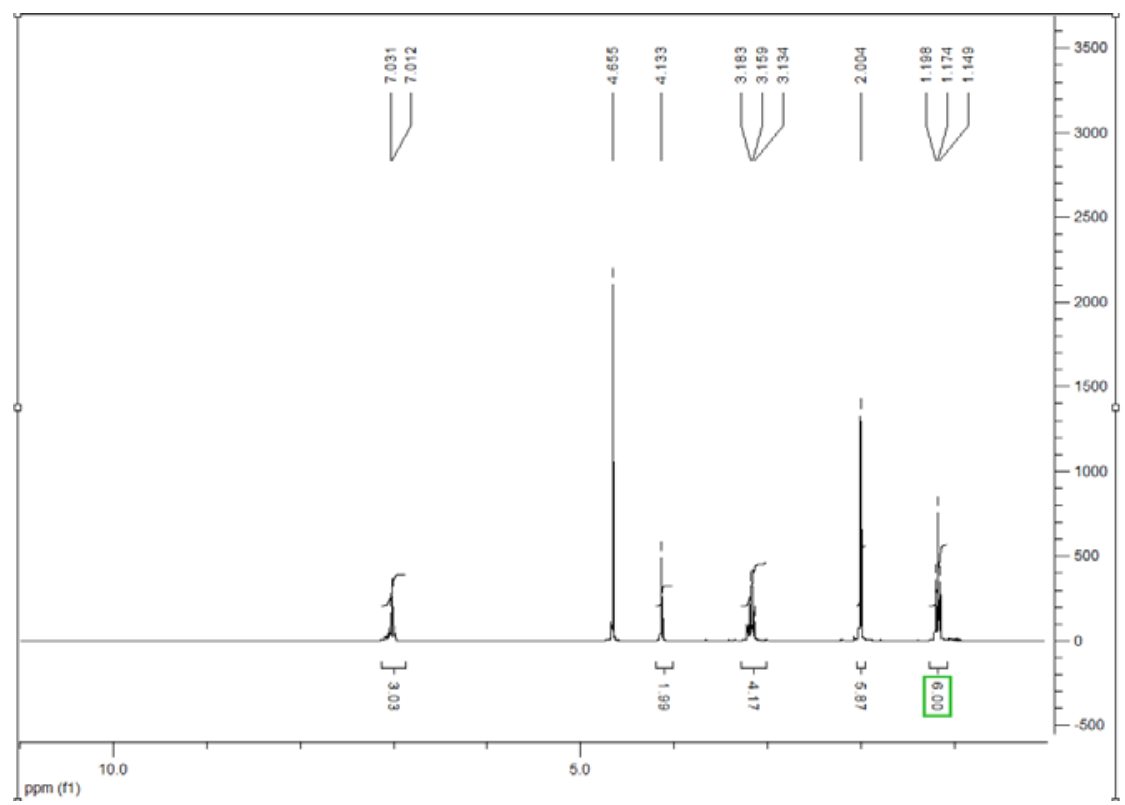

Figure S14. ${ }^{1} \mathrm{H}$ NMR $\left(\mathrm{D}_{2} \mathrm{O}\right)$ of purified lidocaine hydrochloride $(5 \cdot \mathrm{HCl})$ after extraction with Method B $\left(\mathrm{NaCl} / \mathrm{NH}_{4} \mathrm{Cl}\right)$. 


\section{High Pressure Liquid Chromatography (HPLC)}

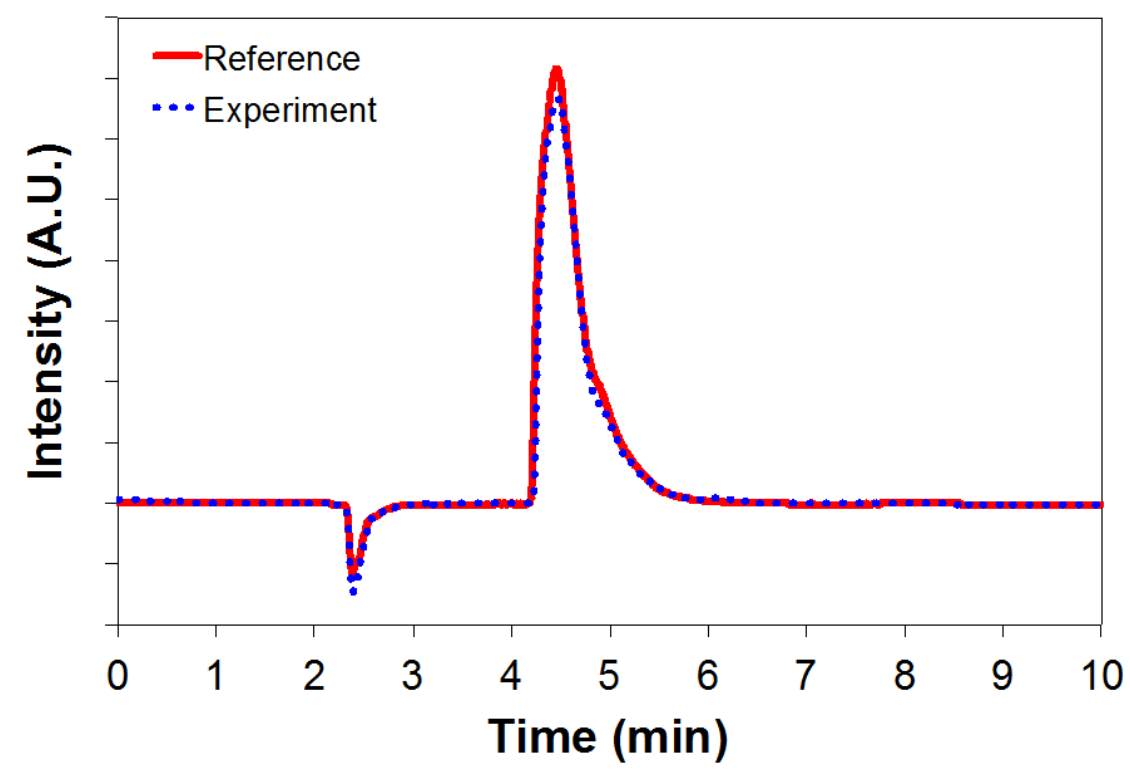

Figure S15. Chromatogram of lidocaine hydrochloride $(5 \cdot \mathrm{HCl})$ produced in the pharmaceutical manufacturing platform applying extraction Method B in upstream (solid blue line) compared to the commercial reference (dashed red line). 


\section{Ultrasound Monitoring}

In-line, real-time concentration and drug release monitoring was conducted with a LiquiSonic 30 system (SensoTech GmbH, Germany). The immersion probe was inducted slightly skewed from the top of the formulation tank (see Figure S4. The ultrasonic standard curve for aqueous formulated lidocaine hydrochloride $(5 \cdot \mathrm{HCl})$ is illustrated in Figure S16.

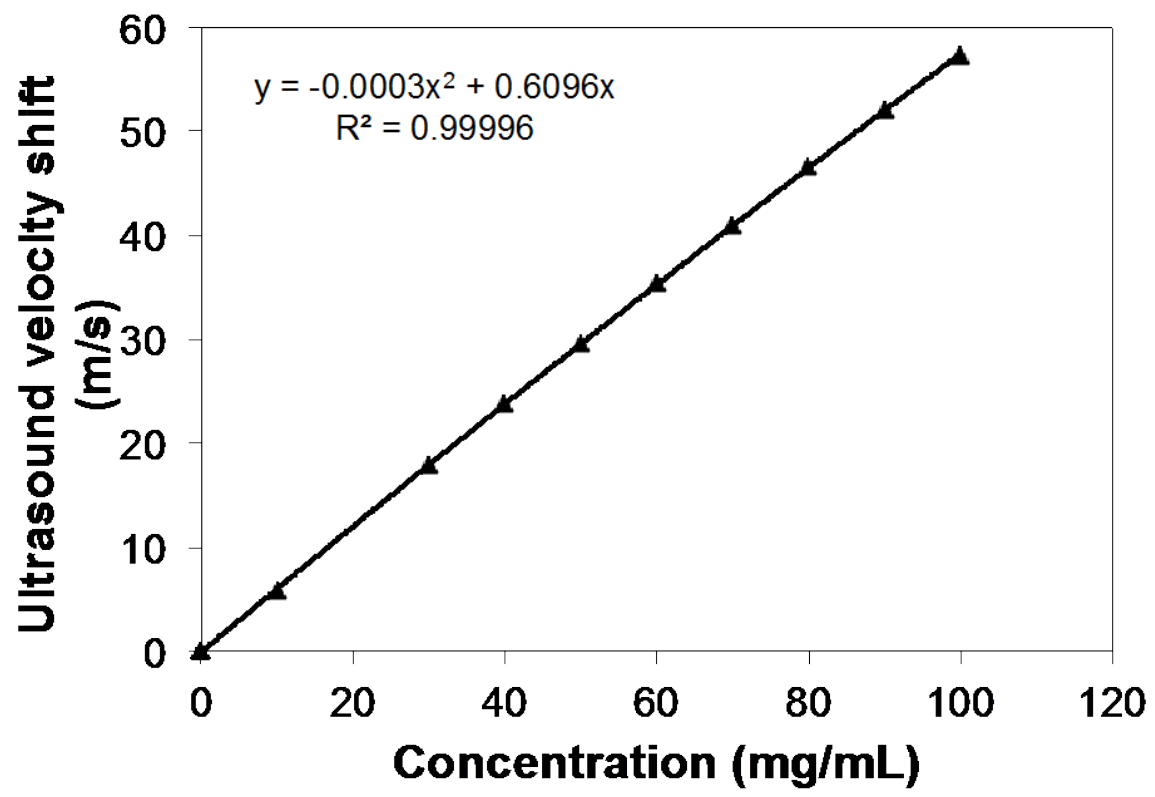

Figure S16. Standard curve of the ultrasonic velocity as a function of lidocaine hydrochloride $(5 \cdot \mathrm{HCl})$ concentration in an aqueous solution of $4 \%$ sodium methylcarboxy cellulose. This curve was used to determine the concentration of $5 \cdot \mathbf{H C l}$ in the formulation tank. 


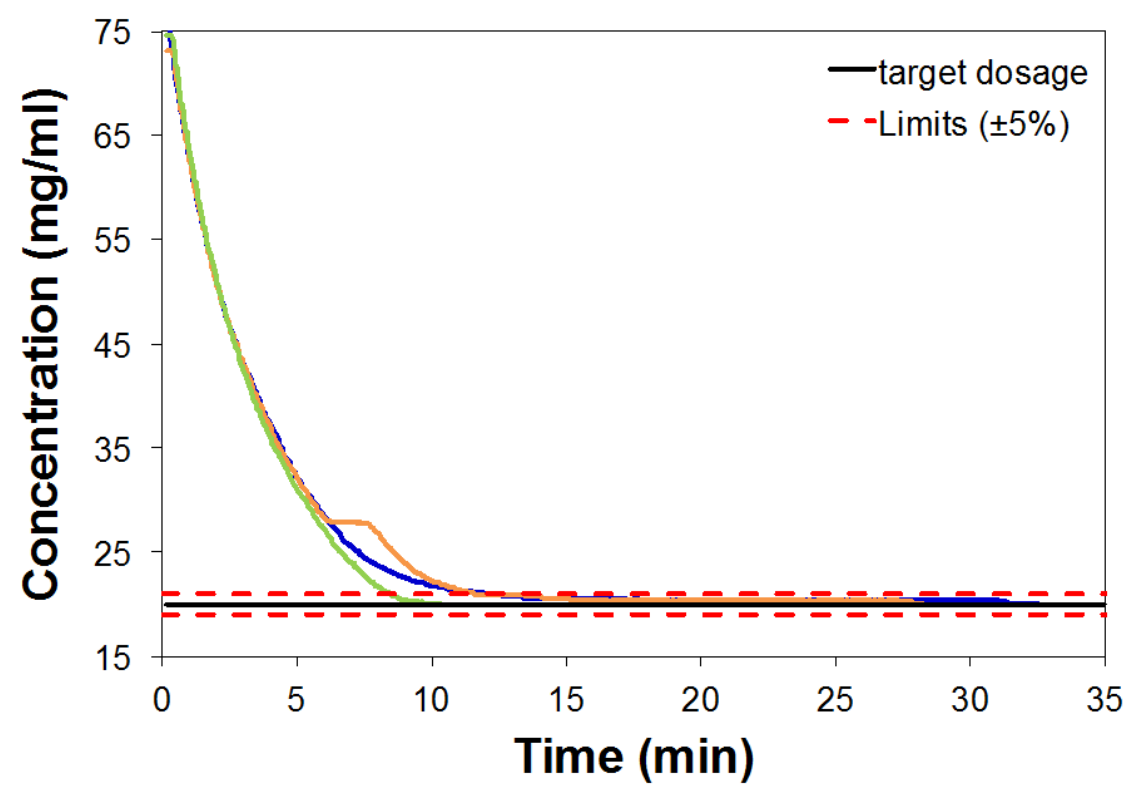

Figure S17. Preliminary studies of concentration adjustments and the real-time, in-situ monitoring of the drug release in the formulation tank for three different PID controller settings. The target dosage of a reference sample (Sigma-Aldrich) of lidocaine hydrochloride $(5 \cdot \mathbf{H C l})$ is $20 \mathrm{mg}$ $\mathrm{mL}^{-1}$ (black solid line); the US Pharmacopeia limits ${ }^{4}( \pm 5 \%)$ are shown as red broken lines. 


\section{Molar Ratio}

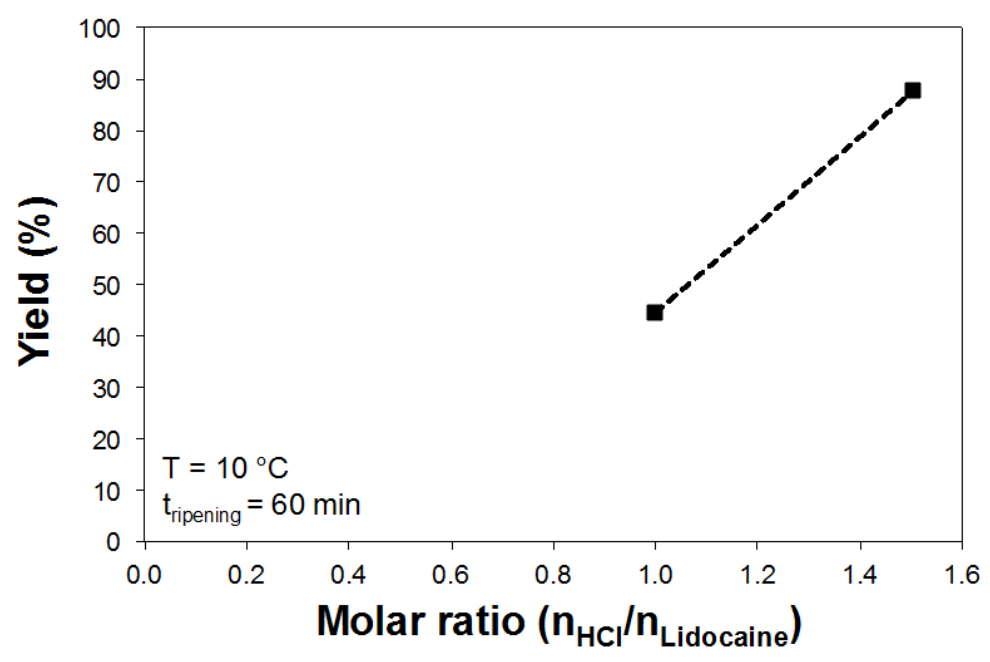

Figure S18. Preliminary studies with commercial lidocaine (5) depicting the improvement in yield by increasing the molar ratio of hydrochloric acid $(\mathrm{HCl})$ to lidocaine (5) from 1.0 to 1.5 at $\mathrm{T}=10^{\circ} \mathrm{C}$ and a ripening time of $60 \mathrm{~min}$ after complete addition of the hydrochloric acid diethyl ether solution $(0.5 \mathrm{M})$.

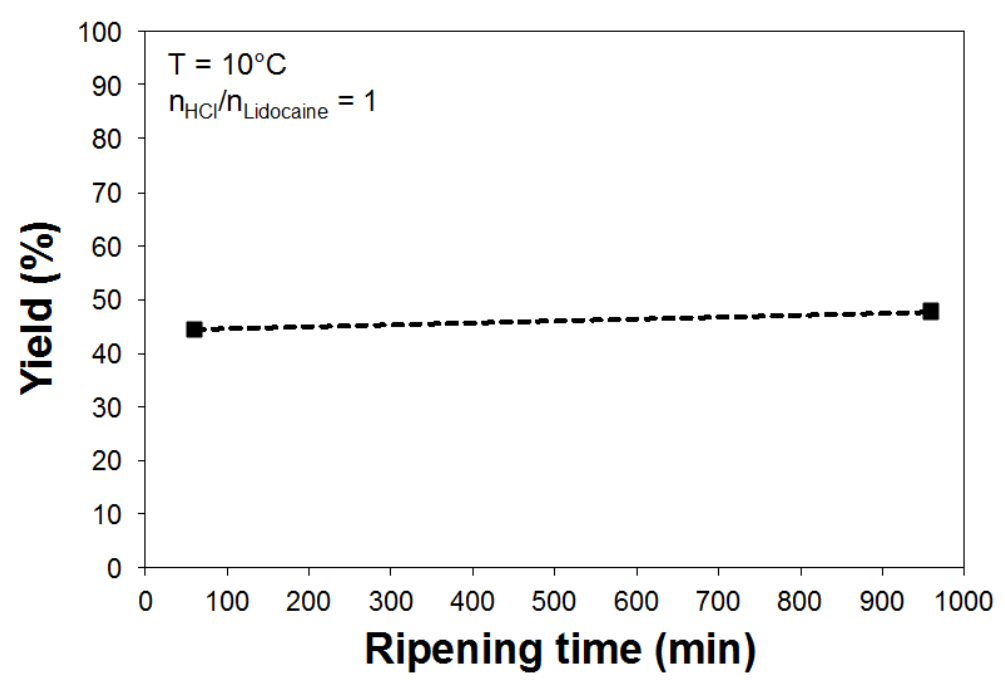

Figure S19. Preliminary studies with commercial lidocaine (5) depicting the improvement in yield by increasing the ripening time of lidocaine hydrochloride $(5 \cdot \mathrm{HCl})$ from $60 \mathrm{~min}$ to $960 \mathrm{~min}$ after complete addition of the hydrochloric acid diethyl ether solution $(0.5 \mathrm{M})$ at a molar ratio of hydrochloric acid ( $\mathrm{HCl}$ ) to lidocaine (5) of 1.0 at $\mathrm{T}=10^{\circ} \mathrm{C}$. 


\section{Additional Experimental Details}

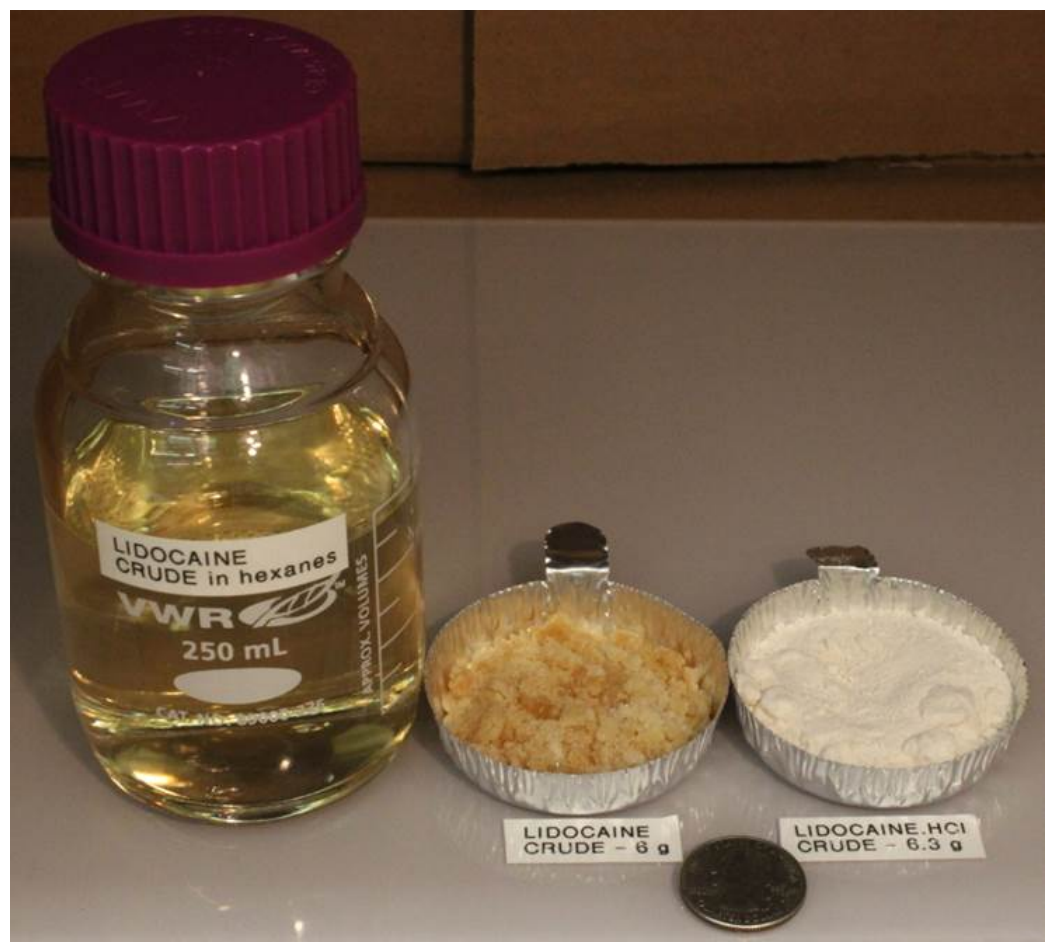

Figure S20. From left to right: photograph of the extracted solution of crude lidocaine (5) collected after $1 \mathrm{~h}$ of operation (hexane); corresponding amount of crude lidocaine (5) obtained upon solvent evaporation and corresponding amount of crude lidocaine hydrochloride $(\mathbf{5} \cdot \mathrm{HCl})$ obtained upon reactive crystallization.

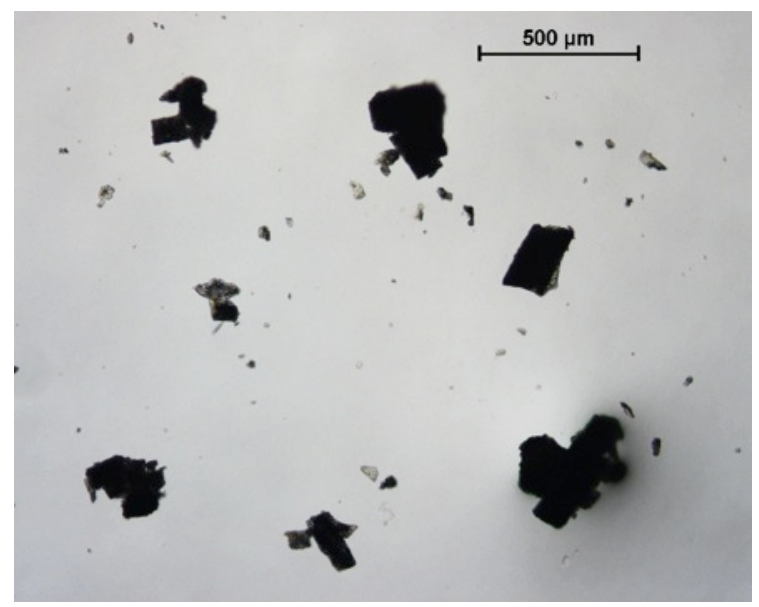

Figure S20. Optical microscope photograph of lidocaine hydrochloride $(5 \cdot \mathrm{HCl})$ obtained by antisolvent cooling crystallization. 


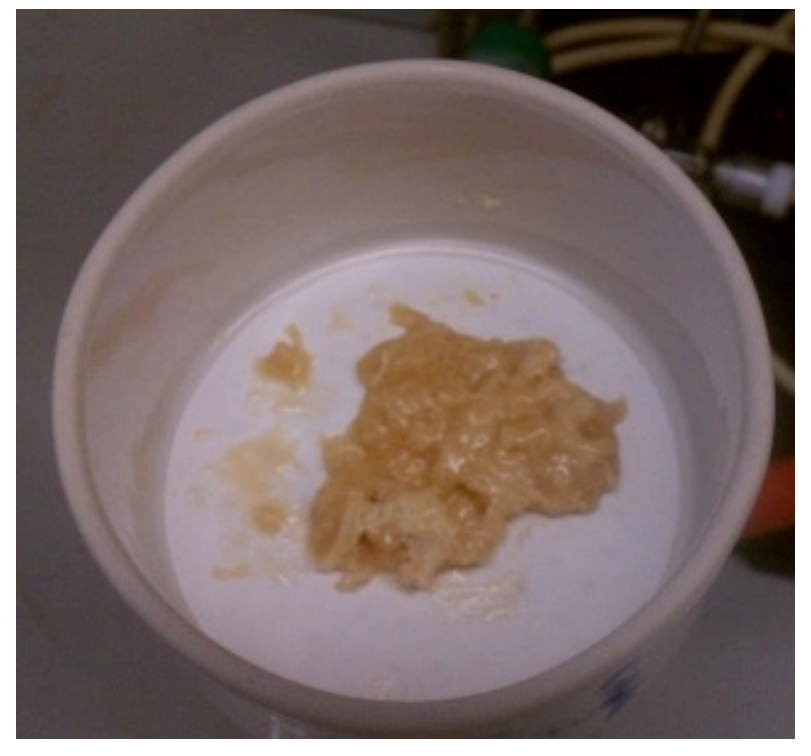

Figure S22. Crude lidocaine hydrochloride $(5 \cdot \mathrm{HCl})$ collected from the reactive crystallization of lidocaine (5) with hydrogen chloride (in diethyl ether) at fast addition rates (Here shown in a Büchner funnel). Under these conditions, crude $5 \cdot \mathrm{HCl}$ crashed out as a sticky material leading to massive incrustation issues in the reaction tank and the subsequent filtration-drying-dissolution unit.

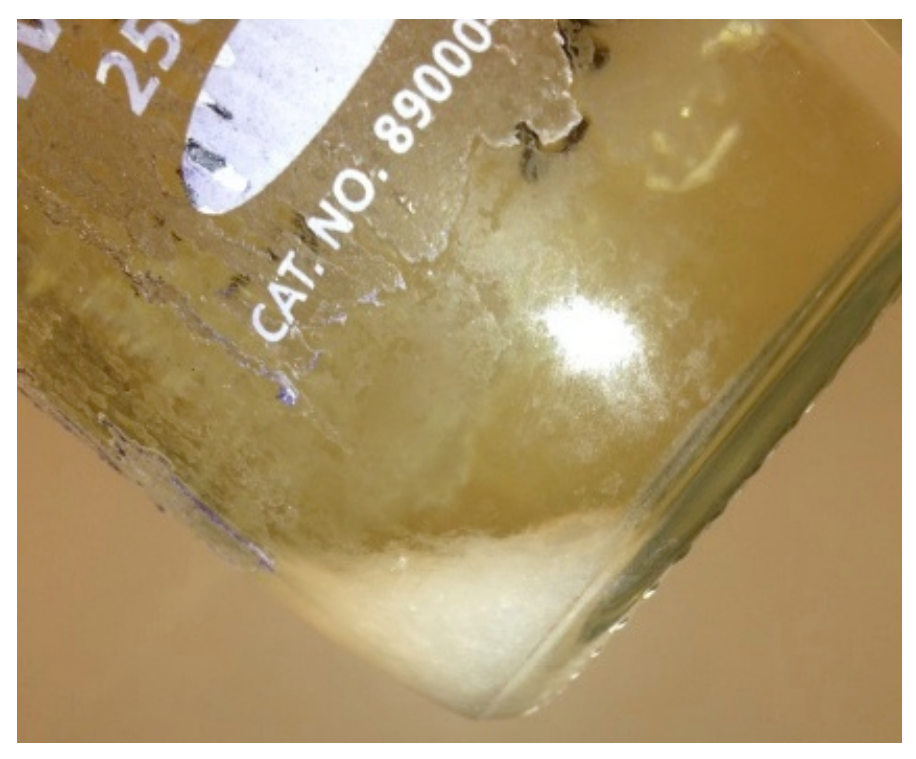

Figure S21. Photograph of crude lidocaine hydrochloride $(5 \cdot \mathrm{HCl})$ contaminated with diethylamine hydrochloride $(\mathbf{4} \cdot \mathrm{HCl})$ produced from extraction Method A. Sample taken from solution 
holding tank. The corresponding crystals are sparingly soluble in an acetone isopropanol (96:4 wt-\%) solvent mixture indicating low content of $5 \cdot \mathbf{H C l}$.

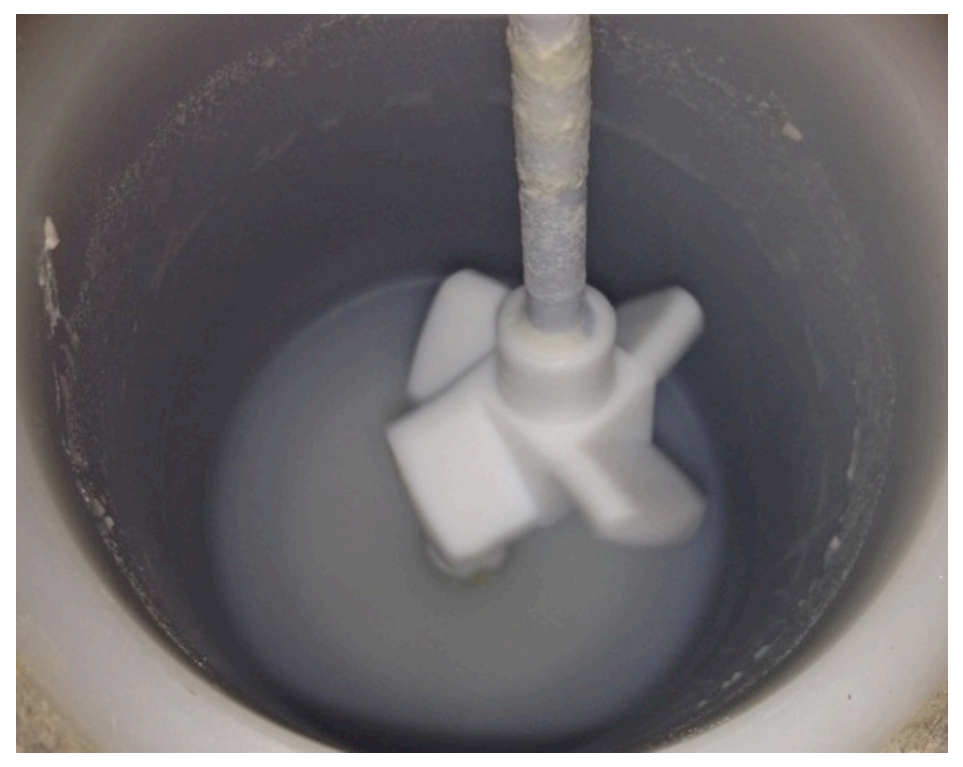

Figure S22. Photograph of the reactive crystallizer after draining of the suspension to the filtration-drying-dissolution unit. Slow addition rate for diethyl ether hydrochloric acid solution led to minor incrustations at the wall and impeller. 


\section{References}

(1) Wong, S. Y.; Chen, J.; Forte, L. E.; Myerson, A. S. Org. Process Res. Dev. 2013, 17, 684692.

(2) Adamo, A.; Beingessner, R. L.; Behnam, M.; Chen, J.; Jamison, T. F.; Jensen, K. F.; Monbaliu, J.-C. M.; Myerson, A. S.; Revalor, E. M.; Snead, D. R.; Stelzer, T.; Weeranoppanant, N.; Wong, S. Y.; Zhang, P. Science, 2016, 352, 61-67.

(3) Nývlt, J. J. Cryst. Growth 1968, 3, 377-383.

(4) USP (US Pharmacopeia). Lidocaine Hydrochloride Oral Topical Solution. Monograph. 2014, pp 3554-3555. 